\title{
ON TORIC VARIETIES AND MODULAR FORMS
}

\author{
PAUL E. GUNNELLS
}

\section{INTRODUCTION}

Let $\ell>1$ be an integer, and consider the congruence subgroup $\Gamma_{1}(\ell) \subset \mathrm{SL}_{2}(\mathbb{Z})$ defined by

$$
\left(\begin{array}{ll}
a & b \\
c & d
\end{array}\right)=\left(\begin{array}{ll}
1 & * \\
0 & 1
\end{array}\right) \bmod \ell
$$

Let $\mathscr{M}_{*}(\ell)=\mathscr{M}_{*}\left(\Gamma_{1}(\ell), \mathbb{C}\right)$ be the ring of holomorphic modular forms on $\Gamma_{1}(\ell)$. In this talk we use the combinatorics of complete toric varieties to construct a subring $\mathscr{T}_{*}(\ell) \subset \mathscr{M}_{*}(\ell)$, the subring of toric modular forms (\$2). This is a natural subring, in the sense that it behaves nicely with respect to natural operations on $\mathscr{M}_{*}(\ell)$ (namely, Hecke operators, Fricke involution, and the theory of oldforms and newforms). Moreover, an explicit structure theorem for $\mathscr{T}_{*}(\ell)$ together with the theory of Manin symbols allows us to describe the cuspidal part of $\mathscr{T}_{*}(\ell)$ in terms of nonvanishing of special values of $L$-functions ( $(3)$. Finally, we discuss an explicit scheme-theoretic embedding of the modular curve $X_{1}(\ell)=\Gamma_{1}(\ell) \backslash \mathfrak{H}^{*}$ in a weighted projective space that was inspired by the structure of $\mathscr{T}_{*}(\ell)(\S 4)$.

The results of $\S \S 2]$ are joint work with Lev Borisov, and can be found in the papers [1, 2, 3]; the embedding of the modular curve in $\$ 4$ is joint work with with Lev Borisov and Sorin Popescu and appears in [4]. It is a pleasure to thank them for their stimulating and interesting collaboration.

\section{Construction And BAsic properties}

Let $d$ be a positive integer, let $N$ be a rank $d$ lattice, let $M$ be the dual lattice, and let $\langle\rangle:, M \times$ $N \rightarrow \mathbb{Z}$ be the pairing. Let $\Sigma \subset N_{\mathbb{R}}=N \otimes \mathbb{R}$ be a complete rational polyhedral fan. A degree function $\operatorname{deg}: N \rightarrow \mathbb{C}$ is a piecewise-linear function that is linear on the cones of $\Sigma$. We define a function $f_{N, \operatorname{deg}}: \mathfrak{H} \rightarrow \mathbb{C}$ by

$$
f_{N, \operatorname{deg}}(q):=\sum_{m \in M}\left(\sum_{C \in \Sigma}(-1)^{\operatorname{codim} C} \text { a.c. }\left(\sum_{n \in C} q^{\langle m, n\rangle} \mathrm{e}^{2 \pi \mathrm{i} \operatorname{deg}(n)}\right)\right) .
$$

Here $q=\mathrm{e}^{2 \pi \mathrm{i} \tau}$, where $\tau \in \mathfrak{H}$, the upper halfplane, and the a.c. denotes analytic continuation.

Theorem 2.1. Suppose deg takes values in $\ell^{-1} \mathbb{Z}$, and that deg is not integral valued on the primitive generator of any 1-cone of $\Sigma$. Then $f_{N, \operatorname{deg}}(q) \in \mathscr{M}_{d}(\ell)$, i.e. $f$ is the $q$-expansion of a holomorphic modular form on $\Gamma_{1}(\ell)$.

To prove this theorem, one begins by showing that this series is well-defined, in the sense that only finitely many terms contribute to a given power of $q$. To prove modularity, let $X_{\Sigma}$ be the toric variety associated to $\Sigma$. Then if $X_{\Sigma}$ is smooth, one uses the Hirzebruch-Riemann-Roch theorem to show

$$
f_{N, \operatorname{deg}}(q)=\int_{X_{\Sigma}} \prod_{D} \frac{(D / 2 \pi \mathrm{i}) \vartheta\left(D / 2 \pi \mathrm{i}-\alpha_{D}, \tau\right) \vartheta^{\prime}(0, \tau)}{\vartheta(D / 2 \pi \mathrm{i}, \tau) \vartheta\left(-\alpha_{D}, \tau\right)}
$$

Date: June 16, 2001.

Partially supported by the NSF. 
where $D$ ranges over the torus-invariant divisors of $X_{\Sigma}, \alpha_{D}$ is the value of deg on the primitive generator of the 1-cone corresponding to $D$, and $\vartheta(z, \tau)$ is Jacobi's theta function. The expression in the integrand is evaluated in the cohomology ring $H^{*}\left(X_{\Sigma}\right)$ using the triple product formula for $\vartheta$. The case of singular $X_{\Sigma}$ is handled using a limiting argument.

Let $\mathscr{T}_{*}(\ell)$ be the full subring of $\mathscr{M}_{*}(\ell)$ given by taking all $\mathbb{C}$-linear combinations of all $f_{N, \text { deg. }}$.

Theorem 2.2. $\mathscr{T}_{*}(\ell)$ is closed under the action of the Hecke operators, the Fricke involution, and Atkin-Lehner lifting.

These statements are proved by direct manipulations of fans and degree functions. For example, if $p$ is a prime not dividing $\ell$, the action of Hecke operator $T_{p}$ on $f_{N, \text { deg }}$ is given by

$$
T_{p} f_{N, \operatorname{deg}}=\frac{p-p^{d-1}}{p-1} f_{N, \operatorname{deg}}+\sum_{S} f_{S, p \operatorname{deg}},
$$

where the sum is taken over lattices $S$ satisfying $N \subset S \subset \frac{1}{p} N$ and $[S: N]=p^{d-1}$. Note that these lattices are not the usual lattices involved in the definition of the Hecke operators.

Remark 2.3. The definition (11) was motivated by L. Borisov and A. Libgober's computation of the elliptic genera of toric varieties [5]. Also, similar sums were studied by W. Nahm, who showed that they had (quasi)modular properties [8].

\section{Special Values of $L$-FunCtions}

A natural question is how far is $\mathscr{T}_{*}(\ell)$ from being all of $\mathscr{M}_{*}(\ell)$. The inclusion $\mathscr{T}_{*}(\ell) \subset \mathscr{M}_{*}(\ell)$ is certainly proper, since for example there are no weight 1 cusp forms in $\mathscr{T}_{1}(\ell)$. However, it turns out that almost all modular forms are toric. To state the precise result, let $\xi=\mathrm{e}^{2 \pi \mathrm{i} / \ell}$, and for $0<a<\ell$ let $s_{a}=s_{a}(q)$ be the weight 1 Eisenstein series

$$
s_{a}(q)=\left.\frac{1}{2 \pi \mathrm{i}} \frac{d}{d z} \ln \vartheta(z, \tau)\right|_{z=a / \ell}=\frac{\xi^{a}+1}{2\left(\xi^{a}-1\right)}-\sum_{d} q^{d} \sum_{k \mid d}\left(\xi^{k a}-\xi^{-k a}\right) .
$$

For each $k$, let $\mathscr{M}_{k}(\ell)=\mathscr{S}_{k}(\ell) \oplus \mathscr{E}_{k}(\ell)$ be the decomposition into cusp forms and Eisenstein series. Given a Hecke eigenform $f$, let $L(f, s)$ be the associated $L$-function.

Theorem 3.1. The ring $\mathscr{T}_{*}(\ell)$ is multiplicatively generated by the $s_{a}, 0<a<\ell$. In weight two, $\mathscr{T}_{2}(\ell)$ modulo $\mathscr{E}_{2}(\ell)$ is equal to the $\mathbb{C}$-span of all Hecke eigenforms $f$ with $L(f, 1) \neq 0$. For weights $k \geq 3$, the space $\mathscr{T}_{k}(\ell)$ coincides with $\mathscr{M}_{k}(\ell)$ modulo $\mathscr{E}_{k}(\ell)$.

In other words, the cuspidal part of $\mathscr{T}_{k}(\ell)$ is easy to describe: for weights $\geq 3$ all cusp forms are toric, and for weight 2 only those in the span of the "analytic rank 0" forms are toric. In general, however, it is not clear what Eisenstein series are toric. For example, in weight 2 the space $\mathscr{T}_{2}(25) \cap \mathscr{E}_{2}(25)$ has codimension one in $\mathscr{E}_{2}(25)$.

The proof of this theorem is a computation with Manin symbols [7]. The space $M(\ell)$ of Manin symbols of level $\ell$ is the $\mathbb{C}$-vector space generated by the symbols

$$
\left\{(a, b) \in(\mathbb{Z} / \ell \mathbb{Z})^{2} \mid \mathbb{Z} a+\mathbb{Z} b=\mathbb{Z} / \ell \mathbb{Z}\right\},
$$

modulo certain relations. This space is dual to the space $\mathscr{S}_{2}(\ell)$ in the following sense. To each symbol $(a, b)$, one can associate an ideal geodesic $\gamma$ on $\mathfrak{H}$. There is an involution on $M(\ell)$ that defines two subspaces $M^{ \pm}(\ell)$. Then there are two subspaces $S^{ \pm}(\ell) \subset M^{ \pm}(\ell)$ such that the pairings $S^{ \pm}(\ell) \times \mathscr{S}_{2}(\ell) \rightarrow \mathbb{C}$ given by integration of a cuspform along a chain are perfect. Moreover the two subspaces $S^{ \pm}(\ell)$ are dual to each other via the intersection pairing on cycles on $X_{1}(\ell)$. 
The key point to the computation is that modulo Eisenstein series the product $s_{a} s_{b}$ has properties similar to the the symbol $(a, b) \in M^{-}(\ell)$. This allows us to define a map $\mu: M^{-} \rightarrow \mathscr{M}_{2}(\ell) / \mathscr{E}_{2}(\ell) \cong$ $\mathscr{S}_{2}(\ell)$ by $(a, b) \mapsto s_{a} s_{b}$. Then we define a map $\varphi: \mathscr{S}_{2}(\ell) \rightarrow \mathscr{S}_{2}(\ell)$ by

$$
f=\sum a_{n} q^{n} \longmapsto \sum a_{n}\left(\int_{0}^{\mathrm{i} \infty}\left(T_{n} f\right) d \tau\right) q^{n},
$$

where $T_{n}$ is the $n$th Hecke operator. This map has the property that $\varphi(f)=0$ if $f$ is a new eigenform with $L(f, 1)=0$. Finally using Merel's universal formula for the Hecke action on Manin symbols, an explicit description of the intersection pairing on $X_{1}(\ell)$, and some manipulations with the product of $q$-expansions $s_{a}(q) s_{b}(q)$, we show that $\varphi$ factors through $\mu$.

For higher weights the argument is similar and its conclusion is identical. We then appeal to a theorem of Jacquet and Shalika [6] that implies $L(f, 1)$ cannot vanish for a new eigenform of weight $\geq 3$.

\section{Equations of modular CURVES}

Let $p \geq 5$ be a prime. Given the simple description of $\mathscr{T}_{*}(p)$ and its relation with the forms of analytic rank 0 , one is interested in estimating $\operatorname{dim} \mathscr{T}_{2}(p)$. As a first step in this direction, we have studied the (incomplete) linear system on the modular curve $X_{1}(p)$ induced the weight 1 Eisenstein series $s_{a}$.

Theorem 4.1. Let $\mathbb{P}$ be the weighted projective space

$$
\operatorname{Proj} \mathbb{C}\left[s_{a}, t_{b} \mid 0<a, b<p\right],
$$

where $\operatorname{deg} s_{a}=1$, and $\operatorname{deg} t_{b}=2$. Then the modular curve $X_{1}(p)$ is scheme-theoretically cut out from $\mathbb{P}$ by the equations (1) $s_{a}=-s_{p-a}$, (2) $t_{b}=t_{p-b}$, and (3) $s_{a} s_{b}+s_{b} s_{c}+s_{c} s_{a}=t_{a}+t_{b}+t_{c}$ if $a+b+c=0 \bmod p$.

Here the variables $t_{b}$ correspond to certain weight 2 Eisenstein series on $X_{1}(p)$.

To prove this theorem, we first show that the map $X_{1}(p) \rightarrow \mathbb{P}^{(p-3) / 2}$ defined by $\tau \mapsto\left\{s_{a}(\tau)\right\}$ is a closed embedding. Then we construct a system of differential equations

$$
\frac{d r_{a}}{d z}=-\frac{1}{p-2} \sum_{k \neq 0, a} r_{k} r_{a-k}+2 r_{a} s_{a}, \quad a \in(\mathbb{Z} / p \mathbb{Z})^{\times}
$$

that mimics the system satisfied by the set of elliptic functions

$$
z \longmapsto \frac{\vartheta(a / p-z, \tau) \vartheta_{z}(0, \tau)}{\vartheta(-z, \tau) \vartheta(a / p, \tau)}
$$

having poles only along a $p$-torsion subgroup. We construct "standard solutions"

$$
r_{a}(z)=\frac{1}{z}+s_{a}+t_{a} z+\cdots
$$

which satisfy certain quadratic relations. If we define a ring using these relations, we get a ring with the same Hilbert function as the coordinate ring of an elliptic normal curve $C$ of degree $p$ in $\mathbb{P}^{p-1}$. Then we show that $C$ is singular if and only if $C$ is a $p$-gon if and only if the coordinates $\left\{s_{a}, t_{b}\right\}$ correspond to a cusp of $X_{1}(p)$. Finally we show that a deformation of a solution to our system leads to a deformation of the elliptic curve, which leads to an identification of the scheme defined by the equations in the theorem with $X_{1}(p)$. 


\section{REFERENCES}

[1] L. A. Borisov and P. E. Gunnells, Toric modular forms and nonvanishing of L-functions, Crelle's J. (to appear).

[2] _ Toric modular forms of higher weight, preprint (2000).

[3] _ Toric varieties and modular forms, Invent. Math. 144 (2001), no. 2, 297-325.

[4] L. A. Borisov, P. E. Gunnells, and S. Popescu, Elliptic functions and equations of modular curves, Math. Ann. (to appear.

[5] L. A. Borisov and A. Libgober, Elliptic genera of toric varieties and applications to mirror symmetry, Invent. Math. 140 (2000), no. 2, 453-485.

[6] H. Jacquet and J. A. Shalika, A non-vanishing theorem for zeta functions of $G L_{n}$, Invent. Math. 38 (1976/77), no. $1,1-16$.

[7] L. Merel, Universal Fourier expansions of modular forms, On Artin's conjecture for odd 2-dimensional representations, Springer, Berlin, 1994, pp. 59-94.

[8] W. Nahm, Quasimodularity of sums over lattices, private communication, 2001.

Department of Mathematics, Rutgers University, Newark, NJ 07102

E-mail address: gunnells@andromeda.rutgers.edu 